\title{
Chemical evolution of r-process elements in the Draco dwarf spheroidal galaxy
}

\author{
M. N. Ishigaki ${ }^{1}$, T. Tsujimoto ${ }^{2}$, T. Shigeyama ${ }^{3}$ and W. Aoki ${ }^{2}$ \\ ${ }^{1}$ Kavli Institute for the Physics and Mathematics of the Universe, University of Tokyo \\ 5-1-5 Kashiwanoha, Kashiwa, 277-8583, Japan \\ email: miho.ishigaki@ipmu.jp \\ ${ }^{2}$ National Astronomical Observatory of Japan, \\ 2-21-1 Osawa, Mitaka, Tokyo 181-8588, Japan \\ email: taku.tsujimoto@nao.ac.jp \\ ${ }^{3}$ Research center for the early universe, University of Tokyo, \\ Bunkyo-ku, Tokyo, 113-0033, Japan \\ email: shigeyama@resceu.s.u-tokyo.ac.jp
}

\begin{abstract}
A dominant astrophysical site for r-process, which is responsible for producing heavy neutron-capture elements, is unknown. Dwarf spheroidal galaxies around the Milky Way halo provide ideal laboratories to investigate the origin and evolution of r-process elements. We carried out high-resolution spectroscopic observations of three giant stars in the Draco dwarf spheroidal galaxy to estimate their europium abundances. We found that the upper-limits of $[\mathrm{Eu} / \mathrm{H}]$ are very low in the range $[\mathrm{Fe} / \mathrm{H}]<-2$, while this ratio is nearly constant at higher metallicities. This trend is not well reproduced with models which assume that $\mathrm{Eu}$ is produced together with Fe by SNe, and may suggest the contribution from other objects such as neutron-star mergers.
\end{abstract}

Keywords. stars:abundances, galaxies:dwarf, galaxies:individual (Draco)

\section{Motivation}

Heavy neutron-capture elements are mainly originated from r-process. These elements are ubiquitous among very metal-poor stars in the Milky Way halo, which suggests that the r-process has been operating since the early Universe (e.g. Sneden et al. 2008, Roederer et al. 2014). Although the physical conditions required for the r-process are well understood, the corresponding astrophysical sites are still unknown. Theoretical studies suggest that core-collapse supernovae (SNe) and/or mergers neutron-stars (or neutron star-black hole systems) could be possible sites for the r-process (e.g. Wanajo \& Ishimaru 2006). These events are expected to have different enrichment timescales and thus can be tested by observed chemical evolution of r-process elements in old stellar populations.

We carried out high-resolution spectroscopic observations to measure europium (Eu) abundances in three giant stars in the Draco dwarf spheroidal galaxy (dSph), which is known to host an old, metal-poor and relatively simple stellar population and thus is a particularly ideal laboratory to study individual r-process events in the early universe. Europium is almost purely produced in the r-process, with little contamination from s-process (c.f. Ba), and thus provides a clean test for the r-process enrichment in this galaxy.

\section{Results and discussion}

The observations of the three Draco stars were carried out with the High-Dispersion Spectrograph (HDS; Noguchi et al. 2002) mounted on the Subaru telescope during Aug 
Table 1. Summary of the atmospheric parameters and abundances.

\begin{tabular}{cccccc}
\hline & $T_{\text {eff }}$ & $\log g$ & $\xi$ & {$[\mathrm{Fe} / \mathrm{H}]$} & {$[\mathrm{Eu} / \mathrm{H}]$} \\
\hline Irwin 19826 & 4028 & 0.6 & 1.7 & $-1.45 \pm 0.12$ & $-1.33 \pm 0.21$ \\
Irwin 20751 & 4278 & 0.6 & 1.7 & $-2.12 \pm 0.13$ & $<-2.0$ \\
Irwin 21275 & 4406 & 0.7 & 2.3 & $-2.51 \pm 0.09$ & $<-2.1$ \\
\hline
\end{tabular}
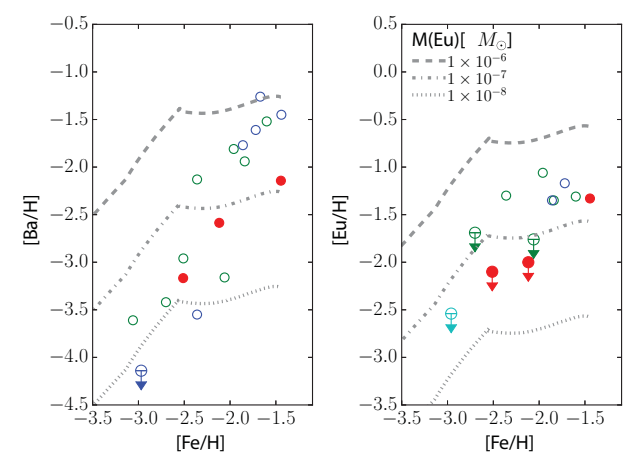

Figure 1 Observed $[\mathrm{Ba} / \mathrm{H}]$ and $[\mathrm{Eu} / \mathrm{H}]$ abundances in this study (filled circles) and those from literature. The gray lines are predictions of chemical evolution models assuming that $\mathrm{Eu}$ is produced in every SNe $\left(10-40 M_{\odot}\right)$ by an amount $10^{-6}, 10^{-7}$, $10^{-8} M_{\odot}$. They are calculated based on the model of Kirby et al. (2011), whose model parameters have been obtained to reproduce observed $\alpha$-elements and $[\mathrm{Fe} / \mathrm{H}]$ distributions in Draco. Mass of $\mathrm{Ba}$, assumed to be produced in $\mathrm{SNe}$, is adopted according to the Eu yields with the r-process ratio $[\mathrm{Ba} / \mathrm{Eu}] \mathrm{r}$-process $=-0.7$. Note that contribution from s-process is not taken into account.

16-18, 2014. An optical wavelength range was covered with a spectral resolution $R \sim$ 30000 .

Results of the Eu measurements are summarized in Table 1. An absorption line of Eu II (6645 $\AA)$ was weakly detected in the most metal-rich star in our sample, Irwin 19826, while only upper limits were obtained for the two more metal-poor stars. Figure 1 shows $[\mathrm{Ba} / \mathrm{H}]$ and $[\mathrm{Eu} / \mathrm{H}]$ abundances plotted against $[\mathrm{Fe} / \mathrm{H}]$ for the sample stars and for stars from literature (Shetrone et al. 2001, Fulbright et al. 2004, Cohen \& Huang 2009). The $[\mathrm{Eu} / \mathrm{H}]$ abundances in the Draco stars are characterized by the very low values at $[\mathrm{Fe} / \mathrm{H}]<-2$, and an apparent plateau $([\mathrm{Eu} / \mathrm{H}] \sim-1.3 \mathrm{dex})$ at higher metallicities. This trend is not well reproduced by canonical chemical evolution models under the simple assumption that $\mathrm{Eu}$ is exclusively produced in SNe together with $\mathrm{Fe}$ (gray lines). These models generally predict a steep increase of $[\mathrm{Eu} / \mathrm{H}]$ with $[\mathrm{Fe} / \mathrm{H}]$ at $[\mathrm{Fe} / \mathrm{H}]<-2$, which becomes shallower at higher metallicities, due to the onset of Type Ia SNe, and thus are unable to simultaneously explain the observed features over the whole $[\mathrm{Fe} / \mathrm{H}]$ range.

This result may indicate that r-process elements were produced, at least in part, in neutron-star mergers (Tsujimoto \& Shigeyama 2014; Tsujimoto et al. 2015). Further observations are needed to test whether similar $[\mathrm{Eu} / \mathrm{H}]-[\mathrm{Fe} / \mathrm{H}]$ trends are observed in other faint dSphs around the Milky Way.

\section{References}

Cohen, J. \& Huang, W. 2009, ApJ, 701, 1053

Fulbright, J. P., Rich, R. M., \& Castro, S. 2004, ApJ, 612, 447

Kirby, E., Cohen, J. G., \& Smith, G. H., et al. 2011, ApJ, 727, 79

Noguchi, K., Aoki, W., Kawanomoto, S., et al. 2002, PASJ, 54, 855

Roederer, I. U., Cowan, J. J., \& Preston, G. W., et al. 2014, MNRAS, 445, 2970

Shetrone, M. D., Côté, P., \& Sargent, W. L. W. 2001, ApJ, 548, 592

Sneden, C., Cowan, J. J., \& Gallino, R. 2008, ARAA, 46, 241

Tsujimoto, T. \& Shigeyama, T. 2014, A\&A, 565, L5

Tsujimoto, T., Ishigaki, M. N., Shigeyama, T., \& Aoki, W. 2015, PASJ, 67, L3

Wanajo, S. \& Ishimaru, Y. 2006, Nuclear Physics A, 777, 676 